\title{
DISCOVERY OF AN ULTRACOMPACT GAMMA-RAY MILLISECOND PULSAR BINARY CANDIDATE
}

\author{
Albert K. H. Kong ${ }^{1}$, RuOlan Jin ${ }^{1}$, T.-C. Yen $^{1}$, C.-P. Hu ${ }^{2}$, C. Y. Hui ${ }^{3}$, P. H. T. TAM ${ }^{1}$, J. TaKata ${ }^{4}$, \\ L. C. C. $\mathrm{LIN}^{1}$, K. S. $\mathrm{CHENG}^{4}$, S. M. PARK ${ }^{3}$, AND C. L. KIM ${ }^{5}$ \\ ${ }^{1}$ Institute of Astronomy and Department of Physics, National Tsing Hua University, Hsinchu 30013, Taiwan; akong @ phys.nthu.edu.tw \\ ${ }^{2}$ Graduate Institute of Astronomy, National Central University, Jhongli 32001, Taiwan \\ ${ }^{3}$ Department of Astronomy and Space Science, Chungnam National University, Daejeon, Korea \\ ${ }^{4}$ Department of Physics, University of Hong Kong, Pokfulam Road, Hong Kong \\ 5 Department of Physics and Astronomy, Seoul National University, Korea \\ Received 2014 August 21; accepted 2014 September 20; published 2014 October 6
}

\begin{abstract}
We report multi-wavelength observations of the unidentified Fermi object 2FGL J1653.6-0159. With the help of high-resolution X-ray observations, we have identified an X-ray and optical counterpart to 2FGL J1653.6-0159. The source exhibits a periodic modulation of 75 minutes in the optical and possibly also in the X-ray. We suggest that 2FGL J1653.6-0159 is a compact binary system with an orbital period of 75 minutes. Combining the gamma-ray and X-ray properties, 2FGL J1653.6-0159 is potentially a black-widow-/redback-type gamma-ray millisecond pulsar (MSP). The optical and X-ray light curve profiles show that the companion is mildly heated by the high-energy emission and that the X-rays are from intrabinary shock. Although no radio pulsation has yet been detected, we estimated that the spin period of the MSP is $\sim 2 \mathrm{~ms}$ based on a theoretical model. If pulsation can be confirmed in the future, 2FGL J1653.6-0159 will become the first ultracompact rotation-powered MSP.
\end{abstract}

Key words: binaries: close - gamma rays: stars - pulsars: general - X-rays: binaries

Online-only material: color figure

\section{INTRODUCTION}

The Fermi Gamma-ray Space Telescope (Fermi) has revolutionized our understanding of the high-energy universe. In particular, gamma-ray-emitting pulsars are a major population discovered with the Large Area Telescope (LAT) on board Fermi. In the second LAT pulsar catalog, there are 117 gamma-ray pulsars, for which 43 of them are millisecond pulsars (MSPs; Abdo et al. 2013; Ray et al. 2012). MSPs are of particular interest because they represent an important stage in the evolution of compact stars. In recent radio surveys of Fermi-observed MSPs, $>75 \%$ them are found in binary systems (see Abdo et al. 2013 for references) and some of the binary systems have a tight orbit $(<24 \mathrm{hr})$. Based on the radio and optical light curves, a significant amount of intrabinary materials exist in the systems and it is very likely that the gamma-ray radiation from the pulsar and/or the pulsar wind is ablating the companion. Eventually it will evaporate the companion, leaving an isolated MSP. Since the pulsar is destroying its companion, it is called a black widow (if the companion star mass $M_{c}<0.05 M_{\odot}$ ) or redback $\left(M_{c}>0.1 M_{\odot}\right)$ pulsar. With new radio pulsar surveys targeted on Fermi sources, new black widows and redbacks have been found (e.g., Roberts 2013; Ray et al. 2012; Abdo et al. 2013).

Traditionally, MSPs are discovered and studied with radio timing. Indeed, all 43 gamma-ray-emitting MSPs are "radioloud" (Abdo et al. 2013). However, "radio-dim" MSPs have not been identified so far. This may be because gamma-ray emissions from MSPs always accompany radio emissions; the radio beam must be large enough so that an observer can see the radio emission in any geometrical configurations. On the other hand, radio and gamma-ray emission regions can be different and, depending on the geometry, it can result in "radio-dim" MSPs if we miss the radio beam (e.g., Venter et al. 2009). If MSPs are "radio-dim," radio observations may not be able to find them. Alternatively, gamma-ray observations are the best way to identify this class of sources. Interestingly, nearly onethird of the 1873 Fermi gamma-ray sources are still unidentified (Nolan et al. 2012) and they are the best candidates for "radio-dim" MSPs.

To identify suitable targets for investigation, we first selected candidates from the Fermi unidentified source catalog based on three criteria: (1) source variability; (2) high Galactic latitude; and (3) gamma-ray spectral shape. We used the variability index in the Fermi catalog to characterize source variability. For pulsars, we expect that they are steady sources. We also identified potential candidates from the gamma-ray spectra. For gamma-ray pulsars, their gamma-ray spectra are usually described by a power law plus an exponential cutoff model (Abdo et al. 2010). We selected sources that are not well fitted with a power-law (PL) model as shown in the catalog. We have carried out a multi-wavelength campaign to search for such objects (Kong et al. 2012; C. Y. Hui et al. 2014, in preparation) and identified the first "radio-dim" MSP candidate 2FGL J2339.6-0532 (Kong et al. 2011; Romani \& Shaw 2011; Kong et al. 2012). However, more recently, radio and gamma-ray pulsation (Ray et al. 2014) as well as radio continuum emission (Kong et al. 2013) were discovered and the source is no longer a "radio-dim" MSP.

In this Letter, we report a multi-wavelength identification of a "radio-dim" gamma-ray MSP candidate that could be associated with an ultracompact X-ray binary.

\section{MULTI-WAVELENGTH IDENTIFICATION}

2FGL J1653.6-0159 is one of the bright Fermi LAT sources found in the first three months of Fermi operation (Abdo et al. 2009) and it remains unidentified. In the second Fermi LAT source catalog (2FGL; Nolan et al. 2012), 2FGL J1653.6-0159 has a curvature significance of 5.3, indicating that the spectral shape is significantly curved and indicating a variability index of 17 , which is equivalent to a steady source (see Section 3.3 
for a detailed analysis using $5.8 \mathrm{yr}$ of LAT data). Finally, 2FGL J1653.6-0159 is located at a Galactic latitude of $25^{\circ}$. All these properties indicate that 2FGL J1653.6-0159 is a gamma-ray MSP candidate. Because of these, extensive deep radio timing observations for pulsation searches have been carried out. However, no pulsation has yet been detected (Ray et al. 2012; Barr et al. 2013).

Using the same technique as for searching the X-ray/ optical counterpart of the first "radio-dim" MSP candidate 2FGL J2339.6-0532, we first checked the archival X-ray data to look for possible X-ray counterparts in the Fermi error circle of 2FGL J1653.6-0159. The field of 2FGL J1653.6-0159 was observed with Chandra on 2010 January 24 for $21 \mathrm{ks}$ with ACIS-I. We reprocessed the data with updated calibration files. Within the $95 \%$ Fermi error circle, there is only one relatively bright X-ray source (CXOU J165338.0-015836). The X-ray-togamma-ray flux ratio is about 0.007 while all other Chandra sources in the error circle are much fainter (see Cheung et al. 2012), with their X-ray-to-gamma-ray flux ratios less than $0.02 \%$. Such a low flux ratio is not typical for an X-ray counterpart to a Fermi source.

We tentatively identified CXOU J165338.0-015836 as a possible X-ray counterpart to 2FGL J1653.6-0159. This X-ray source was also listed in Cheung et al. (2012) as a potential X-ray counterpart to 2FGL J1653.6-0159. Within the 90\% error circle of CXOU J165338.0-015836, we identified a $R \sim 20$ star that is 0.44 arcsec from the Chandra position in the SuperCOSMO Sky Survey (Hambly et al. 2001). This is also the same star identified in the USNO catalog (Cheung et al. 2012). Given the positional coincidence, we suspected that this is the optical counterpart to CXOU J165338.0-015836. Based on the X-ray spectral fit from Cheung et al. (2012), CXOU J165338.0-015836 has an unabsorbed $0.5-2 \mathrm{keV}$ flux of $9.5 \times 10^{-14} \mathrm{erg} \mathrm{s}^{-1} \mathrm{~cm}^{-2}$. This yields an X-ray-to-optical flux ratio of about 3, which is too high for a foreground star and is not typical for an active galactic nucleus (e.g., Green et al. 2004; Laird et al. 2009). Instead, the $B-R$ color $(B-R \approx 0.5-1$ based on the USNO catalog) looks like a late-type star. All these are very similar to 2FGL J2339.6-0532 (Romani \& Shaw 2011; Kong et al. 2012). We therefore believe that this X-ray/optical counterpart is associated with 2FGL J1653.6-0159 as a gamma-ray MSP candidate.

We have searched for the NVSS catalog and there is no $1.4 \mathrm{GHz}$ source at the $\mathrm{X}$-ray/radio position. Deep radio timing observations have been conducted to look for pulsation from 2FGL J1653.6-0159 and a flux limit of $70 \mu \mathrm{Jy}(1.36 \mathrm{GHz})$ has been set (Barr et al. 2013).

\section{OBSERVATIONS AND DATA ANALYSIS}

Because 2FGL J1653.6-0159 is a potential "radio-dim" gamma-ray MSP, we have performed an optical follow-up observation for its optical counterpart. We also reanalyzed the Chandra X-ray data and performed a detailed analysis using 5.8 yr of Fermi LAT data.

\subsection{Optical Photometry}

We carried out an optical time series observation for the proposed optical counterpart of 2FGL J1653.6-0159 in the $r^{\prime}$ and $g^{\prime}$ bands with the $2.5 \mathrm{~m}$ Isaac Newton Telescope (INT) and the Wide Field camera (WFC) in La Palma on 2014 June 4-5. The exposure time for all observations is six minutes, lasting for about six hours each night. The WFC was operated in a $5^{\prime} \times 5^{\prime}$ windowing mode with a readout time of about $6 \mathrm{~s}$.
All images are flat-field and bias-corrected and we performed relative photometry by comparing with several comparison stars in the field. The observing time was barycentric-corrected. The light curves clearly show variability on a timescale of $1.2 \mathrm{hr}$ (see Figure 1). We then performed a timing analysis by using the Lomb-Scargle periodogram and a 75 minute periodicity is highly significant in both bands. We then applied an observedminus-calculated (O-C) diagram analysis to obtain a best-fit ephemeris of BJD (2456813.48210 \pm 0.00078$)+(0.051930 \pm$ $\left.3.1 \times 10^{-5}\right) \times N$. The phase 0 is defined as the optical maximum in the $r^{\prime}$ band (superior conjunction; see Section 4). We also show a binned $g^{\prime}-r^{\prime}$ light curve that also exhibits a 75 minute periodicity in Figure 1.

\subsection{Chandra}

The phase-averaged Chandra observation was reported in Cheung et al. (2012) and here we focus on the timing analysis and phase-resolved spectroscopy. A detailed X-ray analysis will be presented in C. Y. Hui et al. (2014, in preparation). Using the optical timing ephemeris above, we applied a barycentric correction to the X-ray photon arrival time and folded the background subtracted $0.3-8 \mathrm{keV}$ light curve. A 75 minute periodicity is suggestive (Figure 1) and is significant at $97 \%$ level based on an $H$ statistic $(H$-value $=8.65)$. A more sensitive $\mathrm{X}$-ray observation is required to confirm this.

To investigate whether the X-ray spectral properties vary at different orbital phases, we have performed a phase-resolved spectroscopy. We have extracted the spectra from two phase ranges that encompass the peak $(\phi=0.0-0.4)$ of the X-ray orbital modulation and the other covers the trough $(\phi=0.5-0.8)$. The background spectra for the corresponding temporal coverage were sampled from a nearby source-free region. The extraction of the source and background as well as the generation of response files were done by using the CIAO script specextract. After background subtraction, there were $\sim 155$ counts and $\sim 72$ counts from the peak and trough intervals in $0.3-8 \mathrm{keV}$, respectively. We grouped the spectra so as to have at least 10 counts per spectral bin.

Since C. Y. Hui et al. (2014, in preparation) has confirmed that the X-rays from 2FGL J1653.6-0159 are non-thermal-dominant, we examined both spectra with an absorbed PL model (see Hui et al. 2014). In view of small photon statistics, we fixed the column absorption at the value inferred from the phaseaveraged analysis, i.e., $N_{\mathrm{H}}=10^{21} \mathrm{~cm}^{-2}$ (see C. Y. Hui et al. 2014 , in preparation). For the peak interval, the best-fit yields a photon index of $\Gamma=1.6 \pm 0.2$ and a normalization of $1.8 \pm 0.3 \times 10^{-5}$ photons $\mathrm{keV}^{-1} \mathrm{~cm}^{-2} \mathrm{~s}^{-1}$ at $1 \mathrm{keV}$. For the trough interval, the corresponding parameters are $\Gamma=1.6 \pm 0.3$ and $8.7_{-1.9}^{+2.1} \times 10^{-6}$ photons $\mathrm{keV}^{-1} \mathrm{~cm}^{-2} \mathrm{~s}^{-1}$ at $1 \mathrm{keV}$. Based on this observation, the photon indices inferred in these two intervals are consistent. We concluded that there is no evidence of X-ray spectral variability across the orbit of 2FGL J1653.60159 found in our investigation. However, in this phase-resolved analysis, the limited photon statistics do not allow us to examine whether or not the spectral feature at $\sim 3.5 \mathrm{keV}$ identified by C. Y. Hui et al. (2014, in preparation) in a phase-averaged analysis exists.

\subsection{Fermi LAT}

The Fermi LAT data used in this work were obtained between 2008 August 4 and 2014 May 30, and are available at the Fermi Science Support Center. ${ }^{6}$ We used the Fermi Science

\footnotetext{
6 http://fermi.gsfc.nasa.gov/ssc/
} 


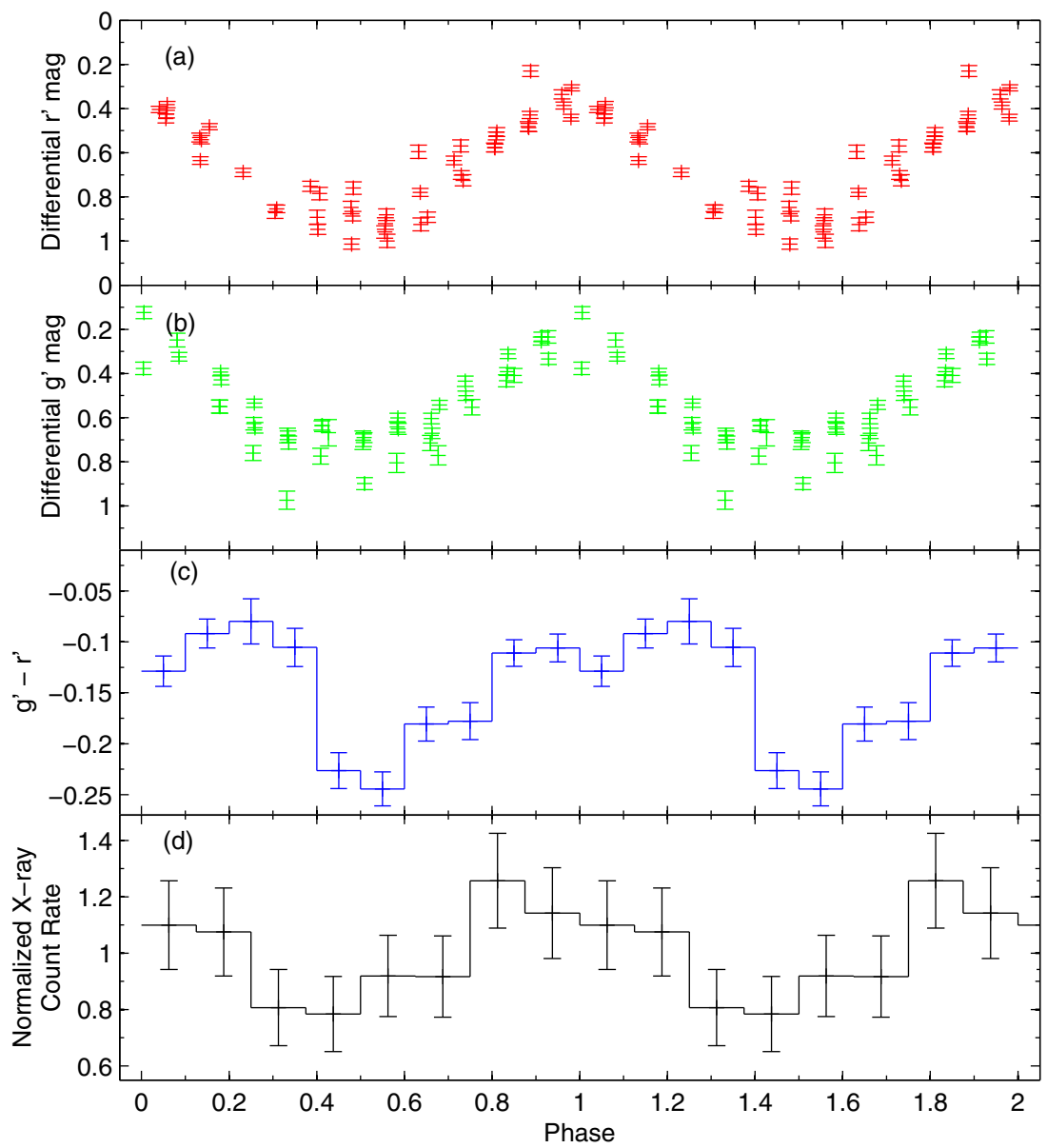

Figure 1. Folded light curve of the $r^{\prime}$ band (a), $g^{\prime}$ band (b), binned $g^{\prime}-r^{\prime}$ (c), and Chandra (d) observations of 2FGL J1653.6-0159 with a best-fit period of 74.7792 minutes. The phase zero is defined as the $r^{\prime}$-band optical maximum (BJD 2456813.48210). It is evident that both the optical and X-ray light curves show similar modulation.

(A color version of this figure is available in the online journal.)

Tools v9r33p0 package to reduce and analyze the data. Only reprocessed pass 7 data classified as "source" events arriving at zenith angles $<100^{\circ}$ were used. The instrument response functions "P7REP_SOURCE_V15" were used.

We carried out a binned maximum-likelihood analysis using gtlike of $0.1-300 \mathrm{GeV}$ events from the rectangular region of $21^{\circ} \times 21^{\circ}$ centered at 2FGL J1653.6-0159. We subtracted the background gamma-ray flux by including the Galactic diffuse model (gll_iem_v05_rev1) and the isotropic background (iso_source_v05), as well as all sources in the second Fermi/LAT catalog (Nolan et al., 2012) within the circular region of $25^{\circ}$ radius around 2FGL J1653.6-0159. The recommended spectral model for each source employed in the catalog was used, while we modeled 2FGL J1653.6-0159 with a simple PL

$$
\frac{d N}{d E}=N_{0}\left(\frac{E}{E_{0}}\right)^{-\Gamma}
$$

and a power law with exponential cutoff (PLE)

$$
\frac{d N}{d E}=N_{0}\left(\frac{E}{E_{0}}\right)^{-\Gamma} \exp \left(-\frac{E}{E_{\mathrm{c}}}\right) .
$$

The normalization values were set free for the Galactic and isotropic diffuse background, as well as sources within $10^{\circ}$ from 2FGL J1653.6-0159.
Based on the difference between - $\log$ (likelihood) values for both models, the PLE model is preferred over the PL model by $10 \sigma$. Using the PLE model, $\Gamma=-1.7 \pm 0.1$, $E_{\mathrm{c}}=3.3 \pm 0.5 \mathrm{GeV}$, and a $100 \mathrm{MeV}-300 \mathrm{GeV}$ energy flux of $(3.52 \pm 0.14) \times 10^{-11} \mathrm{erg} \mathrm{cm}^{-2} \mathrm{~s}^{-1}$ are obtained. These spectral parameter values are typical of those found for gammaray MSPs (Abdo et al. 2009). We divided the $100 \mathrm{MeV}-300 \mathrm{GeV}$ gamma-rays into nine energy bins and reconstructed the flux using gtlike for each band separately. The spectrum is shown in Figure 2. For each bin above $10 \mathrm{GeV}$, a PL model with $\Gamma_{\gamma}=3.0$ was assumed for 2FGL J1653.6-0159 to derive the $90 \%$ confidence-level upper limits.

We probed any long-term flux variation by constructing a gamma-ray light curve from 2008 August to 2014 May with a bin size of 90 days. The source model as described above was used to estimate the background using likelihood analysis. No significant variation was seen in the light curve. Folding the gamma-ray photons at the 75 minute orbital period did not reveal any gamma-ray modulation as well. We derived a $3 \sigma$ upper limit of $21 \%$ for the $1-10 \mathrm{GeV}$ fractional variation of the 75 minute modulation (see de Jager 1994).

\section{DISCUSSION}

Using optical, X-ray, and gamma-ray data, we identified the X-ray and optical counterpart to the Fermi unidentified 
Table 1

Physical Parameters of 2FGL J1653.6-0159 and Six Other Representative Black Widow/Redback Systems

\begin{tabular}{lllcccl}
\hline \hline Source & $\begin{array}{c}\mathrm{D} \\
(\mathrm{kpc})\end{array}$ & $\begin{array}{c}P \\
(\mathrm{~ms})\end{array}$ & $\begin{array}{c}L_{\mathrm{sd}} \\
\left(10^{34} \mathrm{erg} \mathrm{s}^{-1}\right)\end{array}$ & $\begin{array}{c}L_{\gamma} \\
\left(10^{33} \mathrm{erg} \mathrm{s}^{-1}\right)\end{array}$ & $\begin{array}{c}L_{X} \\
\left(10^{31} \mathrm{erg} \mathrm{s}^{-1}\right)\end{array}$ & $\begin{array}{c}P_{\text {orb }} \\
(\text { day })\end{array}$ \\
\hline 2FGL J1653.6-0159 & $1^{\mathrm{a}}$ & $?$ & $?$ & 4 & 2 & 0.052 \\
1FGL J2339.7-0531 & 0.7 & 2.88 & 2 & 2 & 4 & 0.19 \\
PSR J1311-3430 & 1.4 & 2.56 & 4.9 & 15 & 5.6 & 0.065 \\
PSR B1957+20 & 2.5 & 1.61 & 10 & 15 & 45 & 0.38 \\
PSR J1023+0038 & 1.3 & 1.69 & 5 & 1.2 & 10 & 0.2 \\
PSR J1723-2837 & 0.75 & 1.86 & 5 & 1 & 7 & 0.62 \\
PSR J2051-0827 & 1 & 4.51 & 0.5 & 0.7 & 0.1 & 0.1 \\
\hline
\end{tabular}

Note. ${ }^{\text {a }}$ Assumed distance.

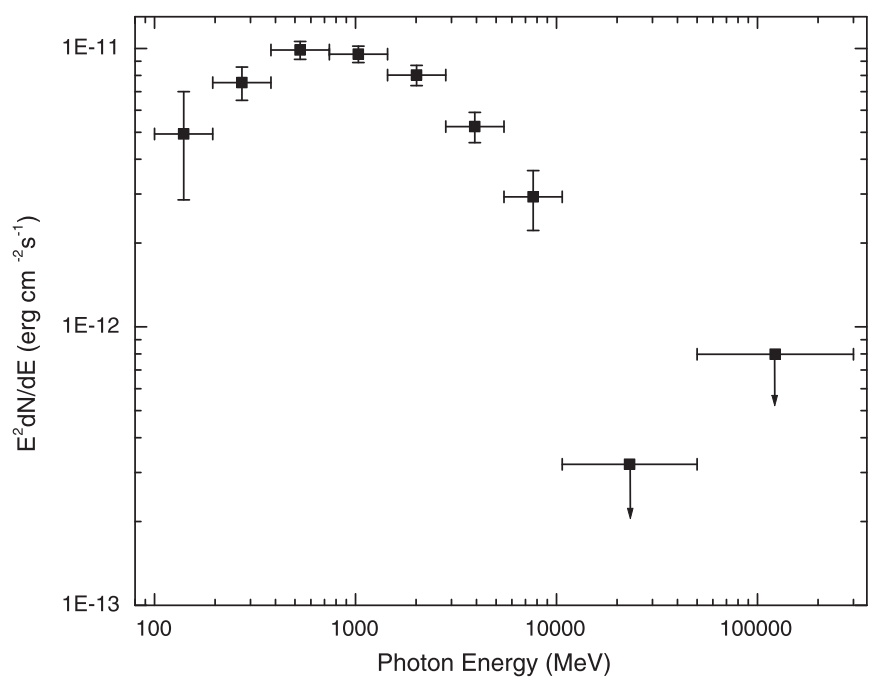

Figure 2. Gamma-ray spectrum derived using 5.8 yr Fermi LAT data (see Section 3.3 for details).

source, 2FGL J1653.6-0159. The most interesting feature is the 74.7792 minute periodicity found in optical data and possibly in X-ray data (see Figure 1). This period can be considered as the binary period of a compact binary system. Given the gamma-ray spectrum and the X-ray-to-gamma-ray flux ratio are consistent with a typical gamma-ray pulsar, we propose that 2FGL J1653.6-0159 is a black widow/redback MSP. Since deep radio timing has not yet found the pulsation (Ray et al. 2012; Barr et al. 2013), 2FGL J1653.6-0159 is very likely a "radio-dim" gamma-ray MSP. This system is very similar to 2FGL J2339.6-0532. While it is no longer a "radio-dim" MSP, it proves that this identification technique can discover MSP candidates from unidentified gamma-ray sources. Using this technique, PSR J1311-3430 was discovered (Romani 2012) and more recently 1FGL J0523.5-2529 was identified as a probable gamma-ray pulsar without a radio counterpart (Strader et al. 2014).

Like 2FGL J2339.6-0532 and other black widows and redbacks, the optical emission of 2FGL J1653.6-0159 is affected by the high-energy heating from the MSP on the companion surface, producing orbital modulation. This indicates that the optical maximum corresponds to the superior conjunction at which the MSP is in between the companion and the observer. We also did a cross-correlation analysis of all light curves and did not find any significant time lag. It is worth noting that the X-ray observation was taken more than four years ago and, given our current uncertainty of the orbital period, a direct comparison may not be correct. Contemporary X-ray/optical observations are required to study the phase alignment of 2FGL J1653.6-0159.

With an observed gamma-ray flux of $F_{\gamma} \sim 3 \times$ $10^{-11} \mathrm{erg} \mathrm{cm}^{-2} \mathrm{~s}^{-1}$ and an assumed typical distance for gammaray MSP of $d \sim 1 \mathrm{kpc}$, the gamma-ray power of 2FGL J1653.60159 is $L_{\gamma}=4 \pi F_{\gamma} d^{2} \sim 4 \times 10^{33} f_{\Omega} d_{1 \mathrm{kpc}}^{2} \operatorname{erg~s}^{-1}$, where $f_{\Omega}$ is the viewing factor. The estimated gamma-ray luminosity $L_{\gamma} \sim 4 \times 10^{33} \mathrm{erg} \mathrm{s}^{-1}$ is a typical value of pulsed gamma-rays from well-known MSPs (see Table 1), and it likely originated from the pulsar magnetosphere. The orbital modulating X-ray emissions will be produced by the intrabinary shock due to the interaction between the pulsar wind and the stellar wind (Kong et al. 2012). The inferred X-ray luminosity from the observed flux is $L_{X} \sim 2 \times 10^{31} d_{1 \mathrm{kpc}}^{2} \mathrm{erg} \mathrm{s}^{-1}$, which is in the range of the observed X-ray luminosity of known black widow/redback systems (see Table 1).

Since the efficiency of the gamma-ray luminosity of MSPs is in general 10\% (Abdo et al. 2013), suggesting the spin down power is of the order of $L_{\mathrm{sd}} \sim 4 \times 10^{34} f_{\Omega} d_{1 \mathrm{kpc}}^{2} \mathrm{erg} \mathrm{s}^{-1}$. Assuming a typical dipole magnetic field of MSPs, $B_{s} \sim 10^{8} \mathrm{G}$, the rotation period is estimated as $P \sim 1.8 \mathrm{~ms}\left(B_{S} / 10^{8} \mathrm{G}\right)^{1 / 2} f_{\Omega}^{-1 / 4} d_{1 \mathrm{kpc}}^{-1 / 2}$ (Takata et al. 2012).

We do not find significant difference between different orbital phase-resolved X-ray spectra. In the context of intrabinary shock between the pulsar wind and the stellar wind from the companion star, this might imply that the orbit is almost circular so that the shock distance from the pulsar has little dependence on the orbital phase. Hence, the spectral properties do not modulate with the orbital phase.

We expect that the optical modulation is caused by the irradiation of the pulsar emissions, indicating the optical peak phase corresponds to the superior conjunction. We also expect that the orbital modulation of the X-ray emission is caused by the Doppler boosting effects of the post-shocked pulsar wind. Since the shift between the optical peak and X-ray peak is less than 0.5 orbital phase, we expect that the shock covers the pulsar, which will be similar to the shock geometry of the redback PSR J1023+0038 (Takata et al. 2014; Li et al. 2014). The nondetection of the gamma-ray orbital period may be because the amplitude of the orbital modulation is too small to be detected by Fermi and/or the inverse-Compoton flux of pulsar wind is smaller than the magnetospheric emissions.

Black widows/redbacks are likely in the late stages of recycling, providing a crucial link between MSPs and accreting millisecond X-ray pulsars (AMXPs). In the family of AMXPs, there are a few ultracompact systems with orbital periods less than 80 minutes (XTE J1751-305, XTE J0929-314, XTE J1807-294, HETE J1900.1-2455, Swift J1756.9-2508, NGC 6440 X-2) and 
therefore ultracompact MSPs should be expected. Indeed, simulations suggest that there should be a large number of ultracompact MSPs in globular clusters (e.g., Rasio et al. 2000). Although the formation of MSPs in the field might be different from those in clusters (e.g., Belczynski \& Taam 2004), some may be formed in clusters and later kicked out to distribute in the field. The striking feature of 2FGL J1653.6-0159 is its very compact binary orbital period (74.7792 minutes) and it may be the first of its kind. If future radio or gamma-ray observations confirm its MSP nature, it will be the most compact rotationpowered MSP binary ever found. Interestingly, the short orbital period can be linked with ultracompact X-ray binaries for which the binary system consists of a compact object (neutron star or black hole) and a degenerate or partially degenerate companion star under a compact ( $<80$ minutes; defined by the period distribution of X-ray binaries) binary orbit (e.g., Nelson et al. 1986; Podsiadlowski et al. 2002). These systems are usually hydrogen-poor objects and if 2FGL J1653.6-0159 is associated with an ultracompact system, the companion is likely a helium star or a white dwarf. Based on binary evolution calculations, 2FGL J1653.6-0159 is more likely a black widow system with a companion much less than $0.1 M_{\odot}$ (Chen et al. 2013). Recently, a new black widow system, PSR J1311-3430, was found to have an orbital period of 94 minutes and the companion is a helium-rich star (Romani et al. 2012). It may be suggestive that the companion star of 2FGL J1653.6-0159 could also be helium-rich similar to ultracompact X-ray binaries. Future optical spectroscopy will confirm this.

Last but not least, pulsation search is required to confirm the MSP nature and gamma-ray pulsation search with LAT will be the best way to try, given that we now have the binary orbital period. At the same time, deep radio imaging will prove whether or not the source is "radio-dim" because imaging observations will not be affected by scattering/absorption due to the intrabinary environment.

Note that another paper by Romani et al. (2014) was submitted slightly earlier than ours and they also identify a similar orbital period. With optical spectroscopy, they found that the companion star is hydrogen-poor.

The INT is operated on the island of La Palma by the Isaac Newton Group in the Spanish Observatorio del Roque de los Muchachos of the Instituto de Astrofísica de Canarias. We thank Diego Torres and Nanda Rea for arranging the INT observations, and Ovidiu Vaduvescu and Teo Mocnik for helping the observing runs. A.K.H.K., R.J., and T.-C.Y. are supported by the Ministry of Science and Technology of the Republic of China (Taiwan) through grants 100-2628-M-007002-MY3, 100-2923-M-007-001-MY3, and 103-2628-M-007003-MY3. J.T. and K.S.C. are supported by a 2014 GRF grant of the Hong Kong government under HKU 17300814P. P.H.T. and C.-P.H. are supported by the Ministry of Science and Technology of the Republic of China (Taiwan) through grants 101-2112-M007-022-MY3 and 102-2112-M-008-020-MY3, respectively. C.Y.H. is supported by the National Research Foundation of Korea through grant 2011-0023383.

Facilities: ING:Newton, CXO, Fermi

\section{REFERENCES}

Abdo, A. A., Ackermann, M., Ajello, M., et al. (Fermi/LAT Collaboration) 2009, Sci, 325, 848

Abdo, A. A., Ackermann, M., Ajello, M., et al. 2010, ApJS, 188, 405

Abdo, A. A., Ajello, M., Allafort, A., et al. 2013, ApJS, 208, 17

Barr, E., Guillemot, L., Champion, D. J., et al. 2013, MNRAS, 429, 1633

Belczynski, K., \& Taam, R. E. 2004, ApJ, 603, 690

Chen, H.-L., Chen, X., Tauris, T. M., \& Han, Z. 2013, ApJ, 775, 27

Cheung, C. C., Donato, D., Gehrels, N., Sokolovsky, K. V., \& Giroletti, M. 2012, ApJ, 756, 33

de Jager, O. C. 1994, ApJ, 436, 239

Green, P. J., Silverman, J. D., Cameron, R. A., et al. 2004, ApJS, 150, 43

Hambly, N. C., MacGillivray, H. T., Read, M. A., et al. 2001, MNRAS, 326, 1279

Hui, C. Y., Tam, P. H. T., Takata, J., et al. 2014, ApJL, 781, L21

Kong, A., Yen, T., \& Fermi Asian Network., 2013, BAAS, 221, 408.06

Kong, A. K. H., Huang, R. H. H., Cheng, K. S., et al. 2012, ApJ, 747, L3

Kong, A. K. H., Huang, R. H. H., Tam, P. H. T., et al. 2011, BAAS, 218, 320.05

Laird, E. S., Nandra, K., Georgakakis, A., et al. 2009, ApJS, 180, 102

Li, K. L., Kong, A. K. H., Takata, J., et al. 2014, ApJ, submitted

Nelson, L. A., Rappaport, S. A., \& Joss, P. C. 1986, ApJ, 304, 231

Nolan, P. L., Abdo, A. A., Ackermann, M., et al. 2012, ApJS, 199, 31

Podsiadlowski, P., Rappaport, S., \& Pfahl, E. D. 2002, ApJ, 565, 1107

Rasio, F. A., Pfahl, E. D., \& Rappaport, S. 2000, ApJL, 532, L47

Ray, P. S., Abdo, A. A., Parent, D., et al. 2012, arXiv:1205.3089

Ray, P. S., Belfiore, A. M., Saz Parkinson, P., et al. 2014, BAAS, 223, 140.07

Roberts, M. S. E. 2013, in IAU Symp. 291, Neutron Stars and Pulsars: Challenges and Opportunities after 80 years, ed. J. van Leeuwen (Cambridge: Cambridge Univ. Press), 127

Romani, R. W. 2012, ApJL, 754, L25

Romani, R. W., Filippenko, A. V., \& Cenko, S. B. 2014, ApJL, 793, L20

Romani, R. W., Filippenko, A. V., Silverman, J. M., et al. 2012, ApJL, 760, L36

Romani, R. W., \& Shaw, M. S. 2011, ApJL, 743, L26

Strader, J., Chomiuk, L., Sonbas, E., et al. 2014, ApJL, 788, L27

Takata, J., Cheng, K. S., \& Taam, R. E. 2012, ApJ, 745, 100

Takata, J., Li, K. L., Leung, G. C. K., et al. 2014, ApJ, 785, 131

Venter, C., Harding, A. K., \& Guillemot, L. 2009, ApJ, 707, 800 